\title{
Discussion on the Operation Mode and Safety Protection of Distribution Live Work
}

\author{
Su Xin, Jin Baoqing, Gao Hui \\ Benxi Power Supply Company of National Grid Liaoning Electric Power Co., Ltd. \\ bxwl_650928@126.com
}

Keywords: distribution line; live work; safety protection; insulation tool

\begin{abstract}
Distribution line is the power infrastructure directly facing the users. With the development of the technology and the improvement of the technical standard in China, it is necessary to further analyze and study the operation mode and safety protection of the distribution live work. This paper discusses the operation mode and the selection of the distribution live work, and puts forward the steps and the safety attentions of the live work.
\end{abstract}

\section{Operation mode of distribution live work}

The operation mode of the distribution live work can be classified by the insulation methods or the insulation tool used.

Classified by insulation methods. According to the insulation methods, it can be divided into indirect and direct operation modes. The indirect operation mode takes the insulation tool as the main insulation and the insulation wearing appliance as the supplementary insulation. The direct operation mode refers to the operators close to the charged body directly by the insulation arm or ladder of work vehicle and work directly by wearing insulation protection apparatus. The distribution live work, whether by a direct method or an indirect method, belongs to the intermediate potential operation mode, if it is divided according to the human body potential of the operators.

Classified by the insulation tool used. The operation modes classified by the insulation tool used are as follows:

(1) Operation mode of insulation tool on the pole. Operators climb up the pole to the appropriate position by means of pole climbing tools, such as climbers, tie the safety belt, maintain a safe distance to the system voltage, and then use the insulating pole whose end is fitted with different tool accessories to do work. The operation mode is not subject to restrictions on traffic and terrain conditions, and it can be operated on the pole which cannot be reached by the aloft insulated boom type aerial.

(2) Operation mode of insulation platform. Insulation platform is usually composed by insulation step ladder, single ladder and insulation vehicle. Operators can work by the indirect method of insulation tools, or by the direct method of insulation gloves, and insulation platform plays a role of the main insulation in phase-to-earth. Whether by indirect or direct methods, before the maintenance phase, the adjacent charged bodies normally should be shielded or isolated by insulation protective and isolated equipment. At the same time, the operators should wear a full set of insulation protective equipment. When they work directly by using the insulation gloves, rubber insulation gloves should be covered by the protective gloves to avoid wearing out or thrusting.

(3) Operation mode of insulated boom type aerial. The insulated boom type aerial of live work was developed in Europe and the United States in 1930s and widely used in the distribution live work in 1950s. The distribution live work carried out by aloft insulated boom type aerial is a convenient, flexible, wide applied operation mode with low labor intensity. 


\section{Selection of operation mode of distribution live work}

The live work of distribution network is different from that of the ultra high voltage transmission line, where the space electric field intensity is high, the operation distance is large, and the operators can enter the high potential and use the direct operation mode to do repair and maintenance by wearing shielding clothing. However, in the distribution live work, due to the low voltage distribution network, small spatial distance between three-phase wires, and intensive distribution facilities, the operation range is narrow, and the human body is easy to touch the power facilities at different potentials. Therefore, the direct operation mode in which the operators wear the shielding clothing and directly contact to the charged body is not suitable for the live work in the distribution network.

Although many units do not have an accident in the application of this mode, it is, strictly speaking, true that there are security risks. On the one hand, it may cause phase to phase short circuit. When the charged body is not covered or not covered completely and the operators are working in the interphase wearing the shielding clothing, they may contact the two-phase charged bodies at the same time if the action range is large. The metal mesh of the shielding clothing will lead to interphase short circuit, and the current of the larger phase short-circuit will get through the shielding clothing, which will not only result in short-circuit of the equipment, but also cause casualties for the short-circuit current exceeding discharge current capacity of the shielding clothing . On the other hand, it may cause a relatively short circuit. When the operators replace insulator and cross arm in line tower and wear a full set of shielding clothing, different parts of their body may also contact the charged body and grounding body and a single-phase ground is formed. Although the $10 \mathrm{kV}$ system is in the way of a neutral un-grounding, if the line is relatively long or connected with a certain length of cable, the current of three-phase capacitor will exceed the discharge current capacity of the shielding clothing as well, causing casualties.

\section{Working environment}

Weather conditions. Distribution live work is not likely to be done in good weather every time, and most of them are carried out in bad weather. However, when the Beaufort force is over 5, humidity is over80, the temperature is below $\mathrm{O}$ degrees Celsius or higher than 38 degrees Celsius and when there is rain, snow, fog, lightning or in some other weather conditions, live work cannot be carried out. These provisions must be followed by the operators of the live work. At the same time, the weather is unpredictable, which possibly brings risks to the operators. Therefore, to prevent the unpredictable situation, the wind instrument and the humidity meter should be equipped in the work field, and the person in charge needs to check the weather conditions frequently, so as to do preparation in advance.

Field environment. Since distribution live work on $10 \mathrm{kV}$ is generally in the prosperous region of the city, the operating environment is complex and diverse, such as traffic and buildings. In addition, $10 \mathrm{kV}$ distribution network is often very complex, and there is no uniform standard for it. Some of the device structure on the line is special, some of the pole switch form is not standardized, and the cross arm distance and arrangement form are not installed as required. And the multi-circuit wires on the same tower are in a variety of forms, and the distance of transmission lines on the same tower of such weak lines as high and low voltage, communication and cable TV is narrow, so that the insulation car is not easy to cross. With the addition of environmental impact, the operators' impatience, the insufficient consideration of these complex factors, and the shortage of practical preventive measures, it may cause casualties. So the operators must carry out a detailed investigation in the field before work and more staff are arranged to manage the traffic complex areas.

Operation steps and safety attentions in the work position. The state of each component should be observed. (1) The detailed examination of the corrosion state of the pole and the root and upper part of the pole racing wire should be done, and prevention measures to avoid collapse should 
be done when necessary; (2) The state of the upper part of the electrical pole should be observed from the ground, (such as circuit number, pole installation, etc.), and rising direction and path of the aloft operating vehicle should be selected.

Basic requirements of electric check: (1) The low voltage line support should be checked by the low-voltage electroscope; (2) The high voltage cross arm should be checked by high voltage electroscope (operators' head should be $30 \mathrm{~cm}$ below the cross arm); (3) If high voltage electroscope displays nothing, low-voltage electroscope should be used for further inspection: (4) The hopper of the aloft operation vehicle should be adjusted, so that the operators' head keeps a safe distance with the charged wire when they stand in the hopper; (5) If operators use climbers to climb up the pole, the safety attentions are the same as the above case.

\section{Technical measures for the safety of live work}

In the actual work, it should always be addressed that once there is bad weather, live work should not be performed. If the live repair has to be done, all technical personnel should be gathered together and the meeting should be organized to propose advices, suggestions and plan of the work program. After the plan and safety measures are in place, the experienced in technical personnel will be selected to do the work. Safety should be put in the first position, and prevention be dominant, so as to prevent the accidents.

Safe distance of live work. The technical data are different from all the systems of possible over voltage in the past. In fact, when the length of the line is not the same, the structure of the system is different, and then the device is different too. In the same working conditions, different lines have different over voltages and the gap is probably great. Because of this, it is necessary to consider the risk of the work rate in the field, so as to calculate the safe distance according to the multiples of actual system voltage. Activities within the minimum safe distance of a live work, including the minimum gap of the live work and the active distance of the operators' body are affected by various factors, including gap of the shape, the voltage of the discharge gap, and polarity changes. Under normal circumstances, the shape of the working gap of the discharge voltage has a significant effect on the safety distance.

Live work with protective gap. The installation of protective gap can improve the safety of live work. The compact multiple lines with boost transformation and turrets window are especially affected largely by the smaller distance, and the traditional practices cannot meet the requirement of the minimum safe distance and protection gap specified by the standard. When the system discharges for over voltage, and the voltage exceeds the working gap, there will be the danger of gap discharge. In order to protect the operators' safety, it is required to set up the protection gap in the field work, so as to protect the possible voltage of gap discharge and the voltage discharged when the gap is smaller than working gap, and avoid the problem of premature protection of the discharge gap voltage, which will limit the voltage range and play a role in protecting the safety of the operators.

Measures to stop the recloser. The recloser is to create opportunities for the voltage generation. Stopping to use the recloser system can effectively reduce the probability of generating voltage, so that the risk of live work is relatively reduced. And it can effectively avoid the secondary damage of the accidents caused by own mistake in the live work, preventing the injuries and the expansion of the accidents.

\section{Conclusion}

With the improvement of the requirements of the reliability of power supply, the distribution live work should be further studied to reduce the range and time of power outage as much as possible on the basis of the program of distribution network live work. Through the research and development of tools, equipment and operation mode, the safety of live work needs to be improved in order to meet the requirements of reliable, stable and economic operation of power network. 


\section{References}

[1]State Grid Corporation of China.The power supply enterprise safety risk assessment standard[M].Beijing:China Electric Power Press,2009.

[2]SAATY $T$ L.Fundamentals of the analytic hierarchy process [M].Pittsburgh:RWS Publications,2000.

[3]DAGDEVIREN M,YUKSEIJ. Developing a fuzzy analytic hierarchy process(AHP)model for behavior—based safety management[J].Information Sciences,2008,178(6):1717- 1733.

[4]JUN Su-ha,SEONG P H.A method for risk-informed safety significance categorization using the analytic hierarchy process and bayesian belief networks[J].Reliability Engineering\&System Safety,2004,83(1):1-15.

[5]PILAVACHI P A,CHATZIPANAGI A I,SPYROPOULOU A I. Evaluation of hydrogen production methods using the analytic hierarchy process[J].International Journal of Hydrogen Energy,2009,34(13):5294-5303.

[6]CHATZIMOURAlrIDIS A I,PILAVACHI P A.Objective and subjective evaluation of power plants and their non- radioactive emissions using the analytic hierarchy process[J].Energy Policy,2007,35(8):4027-4038.

[7]OMASA T,KISHIMOTO M,KAWASE M,et a1.An attempt at decision making in tissue engineering:reactor evaluation using the analytic hierarchy process(AHP) [J].Biochemical Engineering Journal,2004,20(2-3):173-179. 